Bull. Mater. Sci., Vol. 6, No. 6, December 1984, pp. 1069-1074. (C) Printed in India.

\title{
Influence of spin orbit coupling and lanthanide contraction on the exchange interaction in rare earth garnets
}

\author{
C M SRIVASTAVA, C SRINIVASAN and R AIYAR \\ Department of Physics, Indian Institute of Technology, Bombay 400076, India \\ MS received 7 May 1983
}

\begin{abstract}
The exchange constants observed in the rare earth iron garnets, obtained from fitting magnetization and paramagnetic susceptibility as a function of temperature have been analysed in terms of Anderson's theory of superexchange. The transfer integrals and covalency parameters have been obtained for various rare earth ions overlapping with oxygen.
\end{abstract}

Keywords. Exchange constants; superexchange; transfer integrals; covalency parameters; molecular field constants.

\section{Introduction}

In an earlier paper (Srivastava et al 1982) we had reported the simultaneous fitting of saturation magnetisation $\left(M_{s}\right)$ and paramagnetic susceptibility $(\chi)$ for the ferrimagnetic garnets $\mathrm{R}_{3} \mathrm{Fe}_{5} \mathrm{O}_{12}$ where $R$ is yttrium or a trivalent rare earth ion with configuration (Xe) $4 f^{n}(7 \leqslant n \leqslant 13)$. We had used the molecular field constants to determine the exchange constants by means of the equation

$$
J_{i j}=\frac{g_{i} g_{j} \mu_{B}^{2} n_{j} \lambda_{i j}}{2 Z_{i j}}
$$

where $g_{i}, g_{j}$ are the $g$ factors of the sublattices $i$ and $j, \lambda_{i j}$ are the molecular field constants, $n_{j}$ is the number of $j$ ions per unit mole and $Z_{i j}$ is the number of $n-n j$ ions to an ion on ith sublattice. But if one of the ions involved is a rare earth ion then (1) is not the correct expression to convert the molecular field constants to $J_{i j}$. This is because for the rare earths the orbital momentum is not quenched and hence the exchange energy is

$$
E_{i}=-2 n_{j} Z_{i j} J_{i j}\left(g_{i}-1\right) \bar{J}_{i} \cdot \bar{S}_{j} \text {. }
$$

Here, we have assumed that the $i$ th ion is a rare earth and the factor $\left(g_{i}-1\right)$ comes because the rare earth spin has to be projected on $J_{i}$ the angular momentum. In terms of $\lambda_{i j}, E_{i}$ is given by

$$
\begin{aligned}
E_{i} & =-\mu_{i} \cdot \mu_{j}=-g_{i} \bar{J}_{i} \mu_{B} \cdot \lambda_{i j} \bar{M}_{j}, \\
& =-n_{j} g_{i} g_{j} \mu_{B}^{2} n_{j} \lambda_{i j} \bar{J}_{i} \cdot \bar{S}_{j}
\end{aligned}
$$

From (2) and (3) we get

$$
J_{i j}=\frac{g_{i} g_{j} \mu_{B}^{2} n_{j} \lambda_{i j}}{2 Z_{i j}\left(g_{i}-1\right)} \text {. }
$$


Table 1. Exchange constants $\left({ }^{\circ} \mathrm{K}\right)$ in garnets $\left(\mathrm{R}_{3} \mathrm{Fe}_{5} \mathrm{O}_{12}\right)$.

\begin{tabular}{lccc}
\hline & \multicolumn{3}{c}{ Values in ${ }^{\circ} \mathrm{K}$} \\
\hline$R$ & $-J_{\text {ac }}$ & $-J_{d c}$ & $-J_{c c}$ \\
\hline Y & 0 & 0 & 0 \\
Gd & 0.60 & 1.8 & 0 \\
Tb & 1.12 & 2.0 & 0 \\
Dy & 2.97 & 2.97 & -0.9 \\
Ho & 2.64 & 2.80 & 0 \\
Er & 2.70 & 3.40 & 0 \\
Tm & 2.64 & 3.96 & 0 \\
Yb & 21.00 & 16.1 & -0.2 \\
\hline
\end{tabular}

The $-J_{a d},-J_{a d}$ and $-J_{d d}$ values are $6.45,30.40$ and 12.05 respectively in all the cases.

Equation (4) differs from (1) because of the factor $\left(g_{i}-1\right)$ in the denominator. Hence the exchange constants have to be corrected by dividing the values reported earlier (Srivastava et al 1982) by $\left(g_{i}-1\right)$ where $g_{i}$ is the $g$ factor of the rare earth site. These are shown in table 1.

\section{Exchange constants from Anderson's theory}

We give below the calculation of exchange constants from Anderson's theory of superexchange for these garnets. For the exchange between a rare earth and $\mathrm{Fe}^{3+}$ ions in garnets Levy (1966) has shown that if the $Z$ axis for the interaction is chosen as the $\mathrm{RE}-\mathrm{O}^{2}$ - bond direction then the only orbital on the RE ion contributing to the exchange is the one with $m_{l}=0$ (i.e. $f_{z^{3}}$ orbital). Following the same convention we find that the only transfer integral in Anderson's expression for $180^{\circ}$ superexchange interaction is $b_{\sigma \sigma}$ arising from the overlaps of $f_{z^{3}}$ orbital on RE ion and $e_{g}\left(d_{z^{2}}\right)$ on $\mathrm{Fe}^{3+}$ with a $p_{z}$ orbital on the $\mathrm{O}^{2-}$ ion. Similarly the only transfer integral for the $90^{\circ}$ exchange is $b_{n \sigma}$ arising from overlaps of $t_{2 g}$ and $f_{z}$ orbitals with $p_{z}$ orbital on oxygen. Thus we have (ignoring direct overlap of magnetic orbitals)

$$
\begin{aligned}
& J_{i j}=-\frac{1}{4\left(g_{i}-1\right) J_{i} S_{j}} 2 \frac{b_{\sigma \sigma}^{2}}{U} \text { for } 180^{\circ} \text { superexchange } \\
& J_{i j}=-\frac{1}{4\left(g_{i}-1\right) J_{i} s_{j}} 2 \frac{b_{n \sigma}^{2}}{U} \text { for } 90^{\circ} \text { superexchange }
\end{aligned}
$$

In rare earths the spin orbit interaction is very strong compared to the crystal field energy, hence the ground state wavefunction will have admixtures of states with all values of $m_{l}$. However, as explained above only the $m_{l}=0$ state contributes to the superexchange. Hence if we assume that all the $m_{l}$ states have equal probability of being occupied then the probability that the state $m_{l}=0$ has unpaired electron is $1-(n-7) / 7=2-(n / 7)$ where $n$ is the number of electrons in the $4 f$ shell $(n \geqslant 7)$. Using this in (5) and noting that $J_{a c}$ is a predominantly $90^{\circ}$ exchange while $J_{d c}$ is a $180^{\circ}$ 
exchange we get for a $f^{n}-d^{5}$ superexchange interaction

$$
\begin{aligned}
& J_{d c}=-\frac{1}{4\left(g_{i}-1\right) J_{i} S_{j}} 2 \frac{b_{\sigma \sigma}^{2}}{U}\left(2-\frac{n}{7}\right), \\
& J_{a c}=-\frac{1}{4\left(g_{i}-1\right) J_{i} S_{j}} 2 \frac{b_{\pi \sigma}^{2}}{U}\left(2-\frac{n}{7}\right) .
\end{aligned}
$$

The values of the coulomb energy can be estimated by a procedure used by Anderson (1959) to evaluate $U$ for the $3 d$ series transition metal ions. Taking the free ion ionisation potential (IP) for these ions we find that $I_{4}-I_{3}$ i.e. the difference between the 4th and 3rd IP gives the free ion $U$. This has to be corrected in the solid. Firstly the transfer of electrons take place through a finite distance and not to infinity as is the case for calculating IP. Anderson estimated this correction to be $4 \mathrm{eV}$ for $3 d$ electrons. We shall assume the same correction for $R^{3+}$ ions. The next correction is the dielectric polarisation correction because the coulomb repulsion takes place in a dielectric medium and not in vacuum. This gives another correction of $4 \mathrm{eV}$. Finally because of covalency another $10 \%$ correction is required. Using the IP calculated by Sugar and Reader (1973) and using the above method we get $U=10 \mathrm{eV} \pm 1 \mathrm{eV}$ for all the rare earth ions except $\mathrm{Gd}^{3+}$ as shown in table 2. Since the ground state of $\mathrm{Gd}^{2+}$ is $5 d^{2} f^{7}$ and not $f^{8}$, the $U$ value for Gd will reduce if the correct IP for $4 f^{7} f^{8}$ transition is taken Hence we can assume $U=10 \mathrm{eV}$ for all the heavy rare earths. Using (4) instead of (1) as was used in our earlier work (Srivastava et al 1982) to get the correct values of $J_{i j}$ we have obtained the values of $b_{\sigma \sigma}$ and $b_{\pi \sigma}$ using (7) for all these garnets which are given in table 3 . In table 3 we also give the distance of closest approach of the rare earth and oxygen orbitals

$$
S^{-1}=\frac{r_{R-o}-\left(r\left(R^{3+}\right)+r\left(\mathrm{O}^{2-}\right)\right)}{r\left(R^{3+}\right)+r\left(\mathrm{O}^{2-}\right)} .
$$

The values of $r_{R-0}, r\left(R^{3+}\right), r\left(\mathrm{O}^{2-}\right)$ are from Tchéou et al (1970), Khattak and Yang (1979) and CRC handbook (1980) respectively. Figure 1 gives a plot of $b^{2}$ against $S^{-1}$ for both $b_{\sigma \sigma}^{2}$ and $b_{\pi \sigma}^{2}$. This curve resembles the curve obtained by Slater (1953) for metals. However, $b_{a \sigma}^{2}$ does not show the peak in the observed region of overlap. Yb does

\begin{tabular}{|c|c|c|c|c|c|c|}
\hline \multirow[b]{2}{*}{ Ion } & \multicolumn{2}{|c|}{ Ionisation potential (eV) } & \multirow{2}{*}{$\begin{array}{c}\text { Free ion } \\
\begin{array}{c}U=I_{4}-I_{3} \\
(\mathrm{eV})\end{array}\end{array}$} & \multirow{2}{*}{$\begin{array}{c}\text { Corrected } U \\
(\mathrm{eV})\end{array}$} & \multirow{2}{*}{$\begin{array}{c}\text { Covalency } \\
\text { correction } \\
(10 \%)\end{array}$} & \multirow{2}{*}{$\begin{array}{c}\text { Corrected } L \\
\text { in solid } \\
(\mathrm{eV})\end{array}$} \\
\hline & $I_{3}$ & $I_{4}$ & & & & \\
\hline Gd & $20 \cdot 63$ & 44.01 & 23.78 & $15 \cdot 38$ & 1.54 & 13.84 \\
\hline $\mathrm{Tb}$ & 21.91 & $39 \cdot 79$ & $12 \cdot 88$ & 9.88 & 0.99 & 8.89 \\
\hline Dy & 22.79 & $41 \cdot 47$ & 18.68 & $10-68$ & 1.07 & 9.61 \\
\hline Ho & 22.84 & $42 \cdot 48$ & 19.64 & 11.68 & $1 \cdot 17$ & $10 \cdot 57$ \\
\hline $\mathrm{Er}$ & 22.74 & 42.65 & 19.91 & 11.91 & $1 \cdot 19$ & 10.92 \\
\hline $\mathrm{Tm}$ & 23.68 & 42.69 & $19 \cdot 01$ & 11.01 & $1 \cdot 10$ & $9 \cdot 91$ \\
\hline $\mathbf{Y b}$ & 25.03 & 43.74 & 18.71 & 10.71 & 1.07 & 9.64 \\
\hline
\end{tabular}
not fit in the above scheme because as shown by Copland (1970) the $5 p$ shell plays a

Table 2. Coulomb energy $U(\mathrm{eV})$ for rare earth ions.

The correction for $n-n$ and dielectric polarization was 8 in all the cases. 
Table 3. Transfer integrals in garnets $\mathrm{R}_{3} \mathrm{Fe}_{5} \mathrm{O}_{12}$.

\begin{tabular}{|c|c|c|c|c|}
\hline \multirow[b]{2}{*}{$R$} & \multirow[b]{2}{*}{$J_{i}$} & \multirow{2}{*}{$\begin{array}{c}b_{\sigma o}^{2} \\
(\mathrm{eV})^{2}\end{array}$} & \multirow{2}{*}{$\begin{array}{c}b_{\mathrm{xd}}^{2} \\
(\mathrm{eV})^{2}\end{array}$} & $\begin{array}{c}S^{-1} \\
r(R-O)-r\left(R^{3+}\right)-r\left(\mathrm{O}^{2-}\right)\end{array}$ \\
\hline & & & & $r\left(R^{3+}\right)+r\left(\mathrm{O}^{2-}\right)$ \\
\hline Gd & 3.5 & 0.0271 & 0.0091 & 0.016 \\
\hline $\mathrm{Tb}$ & 6.0 & $0-0295$ & 0.0168 & 0.022 \\
\hline Dy & 7.5 & $0-0448$ & 0.0448 & 0.024 \\
\hline Ho & 8.0 & 0.0419 & 0.0398 & 0.026 \\
\hline Er & 7.5 & 0.0513 & 0.0408 & 0.032 \\
\hline $\mathrm{Tm}$ & $6 \cdot 0$ & 0.0596 & 0.0398 & 0.035 \\
\hline $\mathrm{Yb}$ & 3.5 & 0.0240 & $1 \cdot 15$ & 0.037 \\
\hline
\end{tabular}

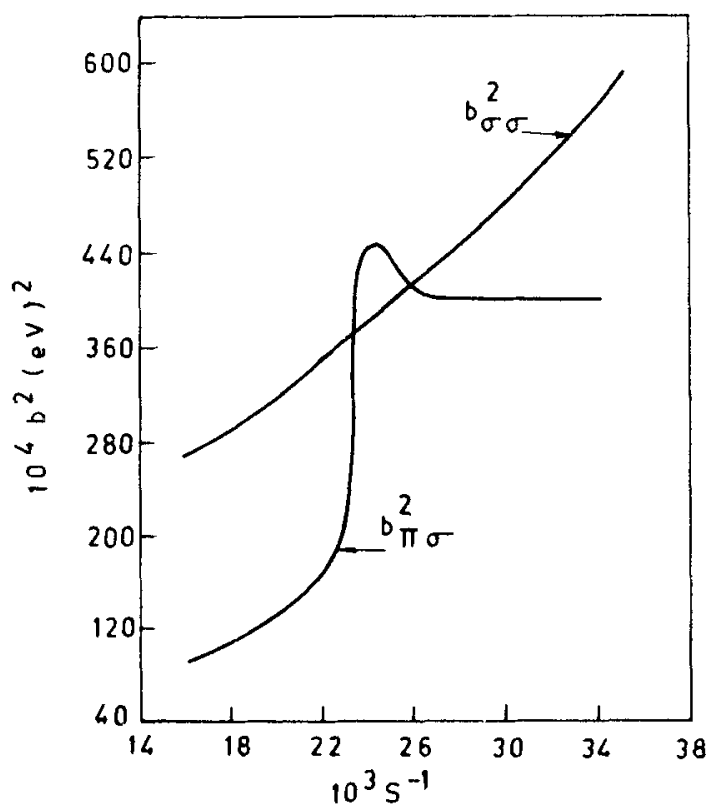

Figure 1. Transfer integrals in garnets.

dominant role in superexchange in $\mathrm{YbIG}$, unlike the other garnets where the $4 f$ shell is the dominant orbital contributing to superexchange.

\section{Covalency parameters}

Zeiger and Pratt (1973) have shown that the transfer integrals $b_{i j}$ are related to the covalency parameters $\lambda_{i c}$ and $\lambda_{j c}$, where $c$ refers to the intervening anion by

$$
b_{i j}=\lambda_{i c} \lambda_{j c} \Delta E_{c}
$$

where $\Delta E_{c}$ is the one electron ionization energy of the anion. For the $\mathrm{Fe}^{3+}-\mathrm{Fe}^{3+}$ 
Table 4. Covalency parameters and transfer integrals in garnets.

\begin{tabular}{|c|c|c|c|c|c|c|}
\hline & $\begin{array}{l}\text { Type of } \\
\text { exchange }\end{array}$ & $\begin{array}{l}\text { Transfer } \\
\text { integral }\end{array}$ & $\begin{array}{l}\text { Value of } \\
b_{k}(\mathrm{eV})\end{array}$ & $\begin{array}{c}\lambda_{a} \\
\left(\mathrm{Fe}^{3+}-\mathrm{O}^{2-}\right)\end{array}$ & $\begin{array}{c}\lambda_{\pi} \\
\left(\mathrm{Fe}^{3+-} \mathrm{O}^{2-}\right)\end{array}$ & $\begin{array}{c}\lambda_{0} \\
\left(R^{3+}-O^{2-}\right)\end{array}$ \\
\hline \multirow[t]{2}{*}{$\mathrm{Fe}^{3+}$} & $J_{a d}$ & $\begin{array}{l}b_{\sigma \sigma} \\
b_{\pi \pi}\end{array}$ & $\begin{array}{l}0.48 \\
0.18\end{array}$ & 0.24 & 0.14 & \\
\hline & $J_{d d}$ & $\begin{array}{l}b_{\sigma \sigma} \\
b_{\pi \sigma}\end{array}$ & $\begin{array}{l}0.12 \\
0.30\end{array}$ & $\begin{array}{l}0.12 \\
0.25\end{array}$ & 0.14 & \\
\hline $\mathrm{Gd}^{3+}$ & $\begin{array}{l}J_{a c} \\
J_{d c}\end{array}$ & $\begin{array}{l}b_{\pi \sigma} \\
b_{\sigma \sigma}\end{array}$ & $\begin{array}{l}0.095 \\
0 \cdot 16\end{array}$ & 0.25 & 0.14 & $\begin{array}{l}0.078 \\
0.08\end{array}$ \\
\hline $\mathbf{T b}^{3+}$ & $\begin{array}{l}J_{a c} \\
J_{d c}\end{array}$ & $\begin{array}{l}b_{\pi \sigma} \\
b_{a \sigma}\end{array}$ & $\begin{array}{l}0.13 \\
0.17\end{array}$ & 0.25 & $0 \cdot 14$ & $\begin{array}{l}0.107 \\
0.08\end{array}$ \\
\hline $\mathrm{Dy}^{3+}$ & $\begin{array}{l}J_{a c} \\
J_{d c}\end{array}$ & $\begin{array}{l}b_{\pi \sigma} \\
b_{\sigma \sigma}\end{array}$ & $\begin{array}{l}0.21 \\
0.21\end{array}$ & 0.25 & $0 \cdot 14$ & $\begin{array}{l}0.17 \\
0.098\end{array}$ \\
\hline $\mathrm{HO}^{3+}$ & $\begin{array}{l}J_{a c} \\
J_{d c}\end{array}$ & $\begin{array}{l}b_{\pi \sigma} \\
b_{\sigma \sigma}\end{array}$ & $\begin{array}{l}0.20 \\
0.20\end{array}$ & 0.25 & $0 \cdot 14$ & $\begin{array}{l}0.16 \\
0.094\end{array}$ \\
\hline $\mathrm{Er}^{3+}$ & $\begin{array}{l}J_{a c} \\
J_{d c}\end{array}$ & $\begin{array}{l}b_{\pi \sigma} \\
b_{\sigma \sigma}\end{array}$ & $\begin{array}{l}0.20 \\
0.22\end{array}$ & 0.25 & 0.14 & $\begin{array}{l}0 \cdot 17 \\
0 \cdot 105\end{array}$ \\
\hline $\mathrm{Tm}^{3+}$ & $\begin{array}{l}J_{a c} \\
J_{d c}\end{array}$ & $\begin{array}{l}b_{\pi \sigma} \\
b_{\sigma \sigma}\end{array}$ & $\begin{array}{l}0.20 \\
0.24\end{array}$ & 0.25 & 0.14 & $\begin{array}{l}0.16 \\
0.11\end{array}$ \\
\hline $\mathrm{Yb}^{3+}$ & $\begin{array}{l}J_{a c} \\
J_{d c}\end{array}$ & $\begin{array}{l}b_{\pi \sigma} \\
b_{\theta \sigma}\end{array}$ & $\begin{array}{l}1.07 \\
0.15\end{array}$ & 0.25 & 0.14 & $\begin{array}{l}0.8 \\
0.07\end{array}$ \\
\hline
\end{tabular}

exchange we have

$$
\begin{aligned}
b_{\sigma \sigma} & =\lambda_{\sigma}\left(\mathrm{Fe}^{3+}\right) \lambda_{\sigma}\left(\mathrm{Fe}^{3+}\right) \Delta E_{c} \\
b_{\pi \pi} & =\lambda_{\pi}\left(\mathrm{Fe}^{3+}\right) \lambda_{\pi}\left(\mathrm{Fe}^{3+}\right) \Delta E_{c} \\
b_{\pi \sigma} & =\lambda_{\pi}\left(\mathrm{Fe}^{3+}\right) \lambda_{\sigma}\left(\mathrm{Fe}^{3+}\right) \Delta E_{c} \\
b_{\sigma \sigma^{\prime}} & =\lambda_{\sigma}\left(\mathrm{Fe}^{3+}\right) \lambda_{\sigma}\left(\mathrm{Fe}^{3+}\right) \Delta E_{c}
\end{aligned}
$$

For $\mathrm{Fe}^{3+}-R^{3+}$ exchange we have

$$
\begin{aligned}
b_{\pi \sigma} & =\lambda_{\sigma}\left(R^{3+}\right) \lambda_{\pi}\left(\mathrm{Fe}^{3+}\right) \Delta E_{c} \\
b_{\sigma \sigma} & =\lambda_{\sigma}\left(R^{3+}\right) \lambda_{\sigma}\left(\mathrm{Fe}^{3+}\right) \Delta E_{c}
\end{aligned}
$$

The electron affinity of oxygen is $-1.47 \mathrm{eV}$ and for $\mathrm{O}^{-}$it is $7.2 \mathrm{eV}$ so that $\Delta E_{\mathrm{c}}=8.67 \mathrm{eV}$. Using this in (7) and (8) we obtain the $\lambda$ values which are listed in table 4 .

We observe that $\lambda_{\sigma}>\lambda_{\pi}$ for $\mathrm{Fe}^{3+}$ exchange as expected from chemical bond theory. Also $\lambda_{\sigma}$ values for the rare earth ions are generally lower than that for the $\mathrm{Fe}^{3+}$ bonds. This also agrees with the chemical theory of covalent bonds where it is known that the covalent bonds involving the $3 d$ orbitals are stronger than those involving the $4 f$ orbitals.

\section{References}


CRC Handbook of Chemistry and Physics 1980-81, 61st (CRC Press Florida) p. F.216

Khattak C P and Yang F F Y 1979 Handbook of physics and chemistry of rare earths (ed.) Schneider Jr and L

Eyrings (Amsterdam: North Holland) Vol. 3 p. 565

Levy P M 1966 Phys. Rev. 147311

Slater J C 1953 Rev. Mod. Phys. 25199

Srivastava C M, Srinivasan C and Aiyar R 1982, J. Appl. Phys. 53781

Sugar J and Reader J 1973 J. Chem. Phys. 592083

Tchéou F, Fuess H and Bertaut H 1970 Solid State Commun. 81745

Zeiger H J and Pratt G W 1973 Magnetic interactions in solids (Oxford: Clarenden) p. 242 